\title{
DALLA TELA ALLA SCENA. ANALISI DRAMMATURGICA DI SHERAZADE VA IN OCCIDENTE DI PATRIZIA MONACO
}

\author{
FROM CANVAS TO THE STAGE. DRAMATUGICAL ANAYSIS OF SHEREZADE \\ VA IN OCCIDENTE BY PATRIZIA MONACO
}

\section{RiAssunto:}

Messi in scena nel 2002, questi cinque atti unici indagano con fine umorismo i clichés culturali che si celano, nel sogno dell'uomo, dietro la rappresentazione delle odalische rinchiuse nei serragli dei sultani. Dalle pièces emerge la capacità dell'autrice di interpretare le opere di grandi maestri della pittura europea (Delacroix, Ingres, Bompard e DebatPonsan), che hanno descritto nei loro quadri la donna araba. Questi test offrono anche ritratti di donne di oggi, consapevoli della loro sensualità e del loro diritto all'amore.

\section{Parole chiave:}

Sherazade va in Oriente, Patrizia Monaco, letteratura e pittura.

\section{Abstract:}

Performed in 2002, this set of five singleact plays constitutes a subtly humorous exploration of the cultural clichés which lurk, in male fantasy, behind the portraits of odalisks enclosed in the harem. These plays demonstrate the author's skill in interpreting the works of the great masters of European painting (Delacroix, Ingres, Bompard e DebatPonsan) who made pictorial representations of Arab women. The texts also offer portraits of women of today, conscious of their own sensuality and right to love.

\section{KeY wORDS:}

Sherazade va in Oriente, Patrizia Monaco, literature and painting. 
Patrizia Monaco, oggetto di un precedente intervento (Trovato, 2012: 1321-1345) è drammaturga attiva e feconda, capace nella sua produzione di attingere fra le diverse forme teatrali quelle che maggiormente le si confacevano per i testo che stava componendo: l'uso del coro della tragedia greca in Tutto per non aver mangiato $i$ cavoletti di Bruxelles, le stazioni del teatro medievale in Una vertigine sopra l'abisso e La porta dell'inferno, il teatro Noh per Ares, il teatro nel teatro in Chi ha paura del padrone cattivo?. In questa relazione analizzerò unicamente cinque atti unici comico-brillanti, satirici e umoristici. Queste briose pièces sono imperniate non tanto sulle ossessioni dell'uomo occidentale riguardo alla donna orientale, quanto piuttosto sul confronto ironico di cinque donne occidentali implicate nel rapporto tra il loro quotidiano e i clichés culturali del sogno esotico dell'Oriente. Ne nascono testi che offrono una graffiante ma nel contempo divertita satira di una società come la nostra votata al culto della bellezza a tutti i costi. Ma insieme i cinque copioni, pur nella loro brevità, sono un invito a sviluppare strumenti di analisi critica tesi a creare rapporti improntati al rispetto reciproco, superando radicati blocchi mentali e steccati ideologici. Pertanto il percorso a carattere interculturale suggerito dalla drammaturga è importante per l'invito al dialogo, alla cooperazione e alla solidarietà da cui i suoi lavori sono innervati e sostanziati.

A quanto annota la drammaturga, “il fascino e l'esotismo delle odalische, delle Sherazade e delle sultane, rinchiuse nei serragli e negli hammam, ritratte in cinque quadri di maestri della pittura europea orientalista ${ }^{1}$, ispirano cinque storie in cui l'Oriente diventa il luogo ideale per l'amore, il sesso, il matrimonio e la bellezza". Quello della Monaco, per riprendere una notazione di una mia allieva (Piastra, 2007: 34), è "un viaggio alla scoperta delle abitudini culturali occidentali, capaci di trasfigurare ogni donna orientale in una potenziale odalisca o Sharazade pronta a scatenarsi in una sinuosa danza del ventre"

Messe in scena col coordinamento registico di Consuelo Barilari², il 9 marzo 2002 nella sala Maestrale dei Magazzini del Cotone al Porto di Genova, queste pièces hanno il titolo complessivo Sharazade va in occidente. In questi pezzi, collocati a metà strada tra modernità e tradizione, l'autrice, oltre a dare con finezza voce a donne che raccontano se stesse, dimostra di sapere interpretare le grandi opere dell'arte orientalistica e nello

1 I quadri ispiratori sono Donne di Algeri nei loro appartamenti di Delacroix, Il bagno turco di Ingres, La grande odalisca, ancora di Ingres, L'attesa di Bompard e Il massaggio di Debat-Ponsan.

2 Dopo aver conseguito il diploma alla Scuola di Recitazione del Teatro Stabile di Genova, lavora con registi come Terry Hands e William Gaskill, con Egisto Marcucci e Marco Sciaccaluga. Si laurea in Filosofia discutendo una tesi sul regista cinematografico Alfred Hitchcock. Entra poi al Teatro della Tosse dove resta come attrice per quindici anni, costruendo le basi per il futuro di regista e manager culturale. Nel 2000 vince il Bando Europeo per la Cooperazione e lo Sviluppo col progetto triennale "Schegge di Nord Africa", kermesse internazionale con i maggiori poeti, letterati, storici e studiosi del Maghreb. Nel 2003 fonda la compagnia "Schegge del Mediterraneo", vincendo il progetto europeo Cultura 2000, classificato tra i primi dieci in Europa. 
specifico quelle di quattro maestri della pittura europea: il romantico Delacroix (17981863), il più accademico Ingres (1870-1867) , Bompard (1857-1936) e Debat-Ponsan (1847-1913) che nell' 800 hanno descritto nei loro quadri la donna araba. I visi velati, tra fascino e mistero, la sensuale atmosfera degli hamman, gli ombrosi cortili vietati agli sguardi degli uomini, turbini di odalische e concubine, harem impenetrabili dai mille segreti, squisite mollezze e profumate stanze, raffinate arti amatorie costituiscono il fascino del Maghreb, universo dai mille segreti. Va detto che i pittori orientalisti, che pure in molti casi non avevano mai visitato l'oriente, ritraevano figure, ambienti, scene di vita del mondo arabo o medio-orientale, carichi di fascino e di esotico mistero e non di rado di una certa sensualità, per la tendenza romantica a vedere nel mondo esotico un ambiente libero dalle convenzioni borghesi occidentali. A quanto ha osservato un giornalista, Paolo Battiflora, su "Il secolo XIX"il 7 marzo 2002, "pittori e avventurieri, viaggiatori e studiosi, letterati e mercanti difficilmente hanno saputo resistere alla malìa di quel mondo arabo, trasfigurato ben presto in esotico mito, in grado di stregare l'immaginario maschile di intere generazioni, dando vita ad un vero e proprio genere artistico, con i suoi bravi stereotipi e clichés". Di qui parte la Monaco per individuare e indagare luoghi comuni, frutto di secolari proiezioni dei colonizzatori europei, su una condizione femminile più immaginaria che reale.

I cinque atti unici sono brevi: il primo è di sei pagine, il secondo di undici, il terzo e il quarto rispettivamente di quattro e dodici e il quinto di cinque, sono intitolati nell'ordine Interior design ovvero Donne di Algeri nei loro appartamenti; Aragosta a colazione ovvero Il bagno turco; Dormire... Sognare ovvero La grande odalisca; Tutto in una notte ovvero L'attesa e Taglia 42 ovvero Il massaggio. Il primo e il secondo testo, rapidi botta e risposta tra due donne, sono stati interpretati uno da Rachele Ghersi ${ }^{3}$ (Giovanna) e Patrizia $\operatorname{Ercole}^{4}$ (Luisa) e l'altro da Federica Granata ${ }^{5}$ (Selina) e Patrizia Ercole (Gloria). Il quarto testo è il contradditorio fra la Barilari (Lisa-Samantha) e AldoVinci ${ }^{6}$ (Silvestro, un

$3 \mathrm{Si}$ è diplomata Attrice presso la Scuola d'Arte Drammatica del Piccolo di Milano. Ha svolto attività radiofonica presso le sedi di Firenze, Milano e Genova. Ha fatto parte di molti spettacoli al teatro Stabile di Bolzano, il Piccolo di Milano e lo Stabile di Genova, lavorando con registi come Scaparro, Squarzina e Sciaccaluga. Insegna dizione alla Scuola di Recitazione dello Stabile del capoluogo ligure.

4 È attrice, regista-pedagoga diplomata allo Stabile di Genova. Laureatasi in Scienze pedagogiche e dell'Educazione, discutendo la tesi su Educare alla teatralità, svolge un'intensa attività di didattica teatrale presso scuole ed enti pubblici e privati Diplomata presso la Scuola di Recitazione dello Stabile genovese, inter.

5 Diplomata presso la Scuola di Recitazione dello Stabile genovese, è fra le attrici de Il gabbiano di Cechov, diretto da Le Moli e prodotto dal Collettivo Due di Parma. Nel 1994, diretta da Cvjetkovic, fa parte del cast di Riccardo III di Shakespeare, prodotto da Teatro Ricerca Salente. Ha girato film ed ha recitato nuovamente a teatro con Pino Petruzzelli.

6 Si diploma alla Scuola di Recitazione dello Stabile di Genova nel 1985. Quasi subito inizia a recitare all'Eliseo di Roma con Lavia, Orsini, Carraro e la Guerritore. Fa cinema e doppiaggio. Tornato a Genova amplia i confini della professione, iniziando un percorso autonomo, caratterizzato 
uomo solo che ha bisogno di uno psicologo). Il terzo e il quinto titolo sono monologhi recitati nell'ordine dalla Granata (Elena), e Simona Guarino ${ }^{7}$ (l'inquieta Lalla). Per allestire i cinque testi è sufficiente un palcoscenico con pochi oggetti: un tavolo da disegno inclinato, una scrivania, due poltroncine e una matita; un letto; una scrivania, due telefoni, un computer e una mensola con alcuni libri, un bollitore per il caffé; un barattolo di nutella, un pacco di fette biscottate, una bottiglia di acqua minerale, alcune mele, dei libri, una spugna e uno specchio.

Nei rapidi pezzi che costituiscono Sharazade vengono offerti con delicatezza ritratti di donne di oggi, consapevoli della loro sensualità e del loro diritto all'amore che non di rado viene loro negato. In queste pièces riaffiorano con chiarezza le componenti dei suoi esordi teatrali: la vena comica e brillante, coniugata con quella satirica e grottesca. I soggetti preferiti della Monaco rimangono le donne, perseguitate e condizionate, di volta in volta, dalla politica, dalla pubblicità e dal consumismo.

Questo copione modulare, costituito cioè da cinque atti unici intercambiabili e usufruibili anche separatamente, nasce su richiesta ${ }^{8}$ di un'attrice, regista e organizzatrice di eventi, la sopra ricordata Barilari, per la festa della Donna del 2002. L'organizzatrice intendeva, afferma l'autrice, "sottolineare l'importanza della civiltà araba che da un anno era negata e derisa dopo l'attentato alle torri gemelle di New York. In giorni in cui ignoranza, paura, malafede facevano trinciare giudizi più o meno insensati e avventati sull'universo islamico, la drammaturga ligure evidenziava per un verso "quanto fosse antica e sontuosa la civiltà islamica" e per l'altro l'esigenza di "distinguere sempre fra estremisti e portatori di una diversa concezione del mondo". Come è noto, dopo il crollo delle Torri Gemelle a New York nel 2001, vi fu nel mondo occidentale una dura reazione che in Occidente mise nello stesso calderone terrorismo e mondo arabo. Di qui la decisione dell'autrice, in sintonia col progetto della Barilari, di "organizzare un evento che da un lato rispondesse a quelle accuse, e dall'altro riguardasse le donne ma fosse stemperato da umorismo e levità".

Il tema specifico elaborato drammaturgicamente dalla Monaco è interessante anche perché basato sullo sguardo di quattro pittori francesi dell' 800 sul mondo arabo e nello specifico quello segreto degli harem. Ne deriva che in questi lavori della Monaco lo sguardo sull'Islam è duplice, filtrato dai pittori orientalisti, ma anche dalla sensibilità odierna.

dall'applicazione dei principi del teatro ad ambiti sociali collaborando con Enti locali, Fondazioni, Ministero della Giustizia e Coop per progetti professionali che coinvolgono minori a rischio, utenti psichiatrici, immigrati, anziani, studenti delle scuole superiori.

7 Diplomatasi allo Stabile, si laurea in Psicologia all'Università di Padova. Oltre che attrice alla radio, al cinema e in televisione, è anche regista.

8 In un'intervista a "Mentelocale" qualche tempo fa la Monaco affermava "Ultimamente, e con questo intendo negli ultimi vent'anni ,sto lavorando molto all'estero e in Italia su commissione". 
In un' intervista la commediografa mi ha detto che, dopo aver avuto come indicazioni registiche le cinque tele sopra ricordate, ha avutola possibilità di sbizzarrirsi liberamente. Poiché sapeva i nomi delle attrici che avrebbero interpretato le pièces ha lavorato nelle condizioni ideali per una drammaturga come lei "che si perita di creare su misura come un sarto". Prima di stenderli la Monaco ha letto, sempre su suggerimento della Barilari, un libro della scrittrice, poetessa, saggista, regista e sceneggiatrice algerina di lingua francese Assia Djebar ${ }^{9}$, intitolato Donne di Algeri nei loro appartamenti ${ }^{10}$, uscito in francese nel 1980 e tradotto in italiano nell' 88 per i tipi della fiorentina Giunti. Quelle pagine, in particolare il capitolo Sguardo vietato, suono tronco, sono state per l'autrice italiana una guida essenziale per penetrare con intelligenza in un mondo che fino ad allora le era del tutto ignoto. In quel volume viene rievocato il viaggio che il pittore Delacroix fece in Marocco e in Algeria nel 1832. Al ritorno in Francia l'artista scrisse lapidariamente :"Dopo il mio viaggio, gli uomini e le cose mi appaiono sotto una luce nuova"11. La Djebar precisa: "Il Marocco si rivela [...] luogo d'incontro tra il sogno e l'ideale estetico fattosi carne, luogo di una rivoluzione visiva"12. Ad Algeri, da dove proveniva, Delacroix aveva visitato un harem, $\mathrm{o}$, per essere più precisi, aveva sbirciato da dietro una tenda un gruppo di donne arabe. Il pittore era inebriato dallo spettacolo che aveva ancora sotto gli occhi: "Là i visitatori sono attesi da un gruppo di donne e di bambini sdraiati in mezzo ad un ammasso di seta e di oro. La sposa dell'ex-rais, giovane e graziosa, è seduta davanti a un narghilè". A quanto riporta la scrittrice franco-algerina, Delacroix "annota per iscritto il particolare che gli sembra più importante: la precisa consistenza dei colori(nero con segmenti dorati, viola laccato, rosso indaco carico ecc.), insieme ai dettagli dei costumi, formano un rapporto molteplice e strano, che sconcerta il suo sguardo"13. L'influenza della scrittrice franco-algerina viene sottolineato dalla Monaco, dal prologo dello spettacolo, con l'ascolto, fuori campo, delle sue parole che, come si legge nel programma di sala, nel prologo ai cinque pezzi, racconta la sua versione della storia della nascita del quadro di Delacroix: "Ad Algeri [..] un rais acconsente a fargli visitare la sua casa, dove il pittore potrà vedere il cuore di un harem, propriamente detto... al suo ritorno a Parigi lavorerà per due anni sull'immagine del suo ricordo, uno stato febbrile e turbato lo trascineranno a scrivere: $\grave{E}$ bello come ai tempi di Omero da questo sentimento confuso nasce il capolavoro che oggi ci costringe a porci delle domande".

9 L'autrice, classe 1936, è la prima maghrebina ad essere stata ammessa nel 2005 all'Accademia di Francia.

10 Il titolo è quello del quadro dipinto dal pittore francese.

11 A. Djebar, cit., p. 159.

12 Ibidem.

13 A. Djebar, cit., pp. 160-161. 
Per capire la mentalità araba, oltre al volume della Djebar, la Monaco ha letto le centoquattordici sure del Corano e riletto le Mille e una notte ${ }^{14}$

Pur condizionata dal richiamo del dipinto di Delacroix, esposto sul palco di fronte al pubblico in una riproduzione su pannello, la commediografa inserisce in un contesto attuale Interior Design. Il titolo da un lato richiama gli appartamenti del titolo del quadro del pittore francese e dall'altro dalla constatazione di quanto per molti sia rassicurante la propria casa. L'interior designer, l'arredatrice d'interni, Giovanna, si sostituisce alla psicologa di cui andava in cerca la protagonista, la ricca Luisa, porgendo con ironia e insieme autoironia i suggerimenti all'altra donna per riconquistare il marito, un politico importante. Una casa arredata come un harem offrirà all'uomo, un fedifrago pigro, una scappatoia. Dal momento che penserà di avere un harem in casa propria, non dovrà più uscire a cercare altre donne. Sebbene l'harem sia composto unicamente dalla propria moglie, l'atmosfera orientale suggerisce a quest'uomo superficiale la sottomissione della donna che gli occidentali sono convinti oggi si sia persa. Per questa ragione vanno a cercare schiave altrove. Non sappiamo se l'uomo si accontenterà e neppure se per la moglie ne varrà la pena. La risposta la darà semmai il pubblico. La battuta finale di Giovanna è: "Come ti dicevo, io vedrei un ampio spazio coperto da folti tappeti e bassi tavolini con narghilè e bracieri da cui scaturiscono intensi profumi. Alle pareti stucchi e pannelli di legno intagliato, broccati a colori che rivestono i divani e tendaggi in seta ricamata che ombreggiano porte e finestre. Nel centro del salone, una vasca incassata nel pavimento. In corrispondenza, un'apertura sul soffitto, da cui filtra morbidamente la luce del sole. Tutto attorno fiori rampicanti, aranci, gelsomini, una nuova Alhambra, gioiello che califfi Omàyyadi di Damasco donarono a Granada...l'ultimo sospiro del Moro"15. Subito dopo, come recita la didascalia, "Luisa si alza e comincia a mimare una danza del ventre". Luisa è una donna chiusa. Pur dichiarandosi una femminista, rivuole tutto per sé il marito per i soldi. Giovanna per contro si dimostra più aperta. Per lei infatti gli uomini sono "come le donne [...] nei sentimenti, nelle emozioni". Tuttavia non è disponibile ad accettare un uomo che neppure la vede.

Nel corso dell'atto unico, scritto al pari dei successivi con grande abilità, assistiamo ad un ribaltamento di ruoli delle due protagoniste. La ristrutturazione degli interni di un appartamento diventa così una metafora di quella degli animi. La Monaco vuole anche dire col suo copione che non dobbiamo crucciarci poi troppo se non siamo quello che sembriamo in quanto ci è concessa la possibilità di vivere con l'immaginazione una bella avventura".

14 L'opera, nella traduzione di Antoine Galland, fatta tra il 1704 e il 1717, ebbe grande notorietà, rappresentando da quel momento "l'esempio più evidente di come l'Oriente sia servito da alter ego all'Occidente che ne costruì una immagine esotica e pittoresca" (M. Cassarino, Prefazione a Le mille e una notte, Torino, Einaudi, 2006, p. XII).

15 La citazione come le successive è tratta dai testi ancora inediti, salvo uno, dell'autrice italiana. 
La seconda pièce, intitolata Aragosta a colazione, vede un gioco a rimpiattino fra due donne sedute su una panca del Louvre. Il titolo del componimento, l'unico ad essere stato pubblicato a cura del Circolo Culturale Brecht di Milano nel 2010 per l'attribuzione alla Monaco del Premio Picasso Sconosciuto, si riferisce all'idea che le donne, appena uscite dal bagno turco, sembrano aragoste cotte. Il quadro di Ingres, capolavoro degli ultimi anni dell'artista, per le nudità di cui è pieno ${ }^{16}$, provoca un evidente turbamento nell'americana Gloria Walker di Madison Wisconsin. In effetti, come si legge nella didascalia che precede la sua battuta iniziale "Comincia ad emettere strani versi, come chi non riesce a respirare bene". Selina Scoubidou ${ }^{17}$, una pied noir parigina, figlia di emigranti dal Maghreb, che di professione, come rivelerà solo nel finale, fa la bibliotecaria, avvertito il disagio della provinciale statunitense, si diverte a prospettarle situazioni personali sempre più spiazzanti, come le affermazioni di essere lesbica, poi di essere da tempo l'amante del di lei marito Frankie, da cui avrebbe avuto due figli; la propria disponibilità a fargliene adottare uno; la dichiarazione di essere una danzatrice del ventre. Ovviamente non è vero nulla di quanto la sua fervida fantasia ha inventato al momento per stuzzicare l'americana. Nel finale ci sarà fra le due un riavvicinamento solidale, quasi di sorellanza, neppure immaginata prima dall'americana. Oramai diventate amiche le due si salutano dandosi un nuovo appuntamento nello stesso luogo. Nel suo lavoro la Monaco, in una battuta pronunciata con una nota irridente da Selina subito dopo una frase della superficiale americana: "E allora viva l'Occidente e le sue certezze incrollabili", vuole dirci che il nostro mondo è fondato su false certezze.

La Monaco mette sulla bocca di Selina la battuta finale nel pirandelliano Così è (se vi pare) detta da quella che non rivelerà se sia la seconda moglie di Ponza oppure la figlia della signora Frola: "Io sono colei che mi si crede"

Il terzo lavoro, Dormire... sognare, è un monologo su "un sogno birichino" e gustoso, mai osceno e volgare, fatto dall' irreprensibile bancaria Elena, che la sera prima aveva letto alcune novelle delle Mille e una notte. Alle spalle del personaggio sta La grande odalisca di Ingres che suscitò, va ricordato, la riprovazione della critica per aver cercato strade nuove spinto dalla mania dell'originalità. Elena, che ha un atteggiamento "di vergogna e autocensura", si immagina di essere una giovane araba che assieme ad altre due, conduce con le parole un piccante gioco amoroso con un focoso giovane che è solamente immaginato Tutte e tre le donne fanno a gara nel trovare nomi sempre

16 Selina afferma che quando Ingres dipinse il quadro accarezza "l'immaginario erotico dell'uomo occidentale vero l'Oriente".

17 I loro due cognomi con tutta probabilità sono riferimenti ironici, il primo al protagonista di una serie televisiva statunitense trasmessa dal 1993 al 2001, Walker Texas Ranger, e il secondo ad un gioco, popolare negli anni '60, di intrecci di fili per fare piccoli oggetti. 
più immaginifici agli organi sessuali maschili e femminili ${ }^{18}$. Elena, abile a cambiare voce, tono e atteggiamenti a seconda delle parti interpretate, si auto-convince di aver mangiato pesante la sera prima, talmente il sogno è vivido e persistente. Il suo può essere definito un sogno interrotto. Al suo risveglio, ad occhi aperti continua a rivivere ciò che aveva solamente immaginato. Nel sogno è presente l'atmosfera del quadro di Ingres riprodotto su un pannello che sta di fronte al pubblico. Per lei si tratta di ricordi di un Oriente che pure non ha mai realmente conosciuto, in cui trovano di continuo sfogo erotismo e simboli ricorrenti nelle Mille e una notte. La bancaria ha sognato qualcosa di cui in fondo avverte con forza l'esigenza, pur provandone vergogna, come prova la sua incapacità di trovare, neppure con se stessa, le parole giuste per dare un nome alla cosa più comune del mondo: fare all'amore con un uomo senza complessi e paure. Nel finale il suo commento disincantato e amaro è: "Ah che comodo sognare quel che non si può vivere. [...] Sognare quel che non si ha il coraggio di vivere [...] Sognare quel che vorremmo essere". In effetti alla fine Elena confessa che non avrebbe mai avuto il coraggio di fare all'amore con il direttore della banca in cui lavora, l'ispettore di Milano, il cassiere, bel moretto maritato, che incontra ogni giorno.

Il quarto copione, Tutto in una notte, le è venuto dal ricordo di un film in cui la donna al di là del filo dell'hot line è una mamma scarmigliata e stremata che, mentre riceve focose telefonate di uomini, accudisce il proprio neonato fra lanci di pappe e sputacchi vari. Nell'atto unico a due voci Lisa-Samantha, giovane attrice per vocazione e telefonista erotica perché disoccupata, si premunisce di un barattolo di nutella, di un pacco di fette biscottate, di una bottiglia di acqua minerale, di alcune mele e di qualche libro per affrontare la sua prima sera di lavoro alla linea telefonica denominata Harem. La protagonista ha a che fare, almeno così crede in un primo momento, con un maniaco che ha il complesso dell'assassino di vergini, come il re Shahriyàr delle Mille e una notte. Silvestro, l'uomo, di cui si sente solo la voce (se ne vedrà il volto quando alla fine esce alla ribalta per gli applausi)., in realtà è un innocuo insonne che, in lunghe e ripetute telefonate a quella che è per lui una comprensiva e paziente interlocutrice, inventa di avere l'impulso irrefrenabile di uccidere giovani donne. Nel finale si scoprirà che è dibattuto in un ambiguo sentimento di amore-morte dovuto alla solitudine. La telefonista, crocerossina del sesso, col complesso di Shahrazàd, vuole con l'arte della parola salvarlo. Le schermaglie tra i due porteranno ad una soluzione positiva. La protagonista sconfigge le ossessioni dello psicopatico che finisce per innamorarsi di lei grazie al fatto di non averla mai vista..

L'ultimo pezzo è un monologo, L'incubo della taglia 42, in cui vengono affrontati temi solo all'apparenza frivoli. Con garbo l'autrice mette alla berlina la stupidità delle donne

18 Non escludo che la Monaco, donna colta , sia stata influenzata dalla lettura di un libro di N. Galli de' Paratesi, Le brutte parole. Semantica dell'eufemismo, edito dal torinese Giappichelli nel 1964 e poi più volte ristampata da Einaudi. 
che vogliono entrare a tutti i costi nella taglia 42, anche se, nel contempo, giustifica la loro legittima aspirazione ad essere guardate e ammirate.

L'inquieta Lalla (il suo vero nome è Maria Paola Tiraboschi), giovane e robusta professoressa di greco e latino, appena uscita dalla doccia, davanti al quadro di DebatPonsin in cui un corpo nudo di donna, affidato alle mani esperte della massaggiatrice crea una forte tensione erotica, è capace di citare con assoluta indifferenza riflessioni del filosofo Kant e banali notazioni che si leggono sulle riviste del parrucchiere. Gli incontri amorosi da lei avuti in passato sono stati vanificati da quelle che la commediografa in un'intervista definisce con termine felice "burroristà". Lalla si rammarica nel finale di non avere imparato l'arabo.

La pièce è l'unica in cui il personaggio interagisce col quadro che sta alle sue spalle: lo guarda, gli parla, in qualche modo fa parte della sua esistenza. La taglia 42 e le su forme rotonde sono la sua ossessione. In questo testo ricorre un'aspra polemica nei confronti del mondo occidentale ("Perché noi che siamo la parte del mondo che affama vuol travestirsi come quella che ha affamato?") ed è manifestata l'esigenza che il Parlamento vari una profonda riforma della scuola che punti anche sulla approfondita conoscenza linguistica e culturale di altre realtà.

Questi atti unici sono ispirati allo sguardo dell' Occidente sull'Oriente in quadri che rappresentano donne nei loro luoghi privati, quali gli harem e gli hammam. Come nei tappeti, le cinque pièces sono percorse dalle varianti degli stessi motivi, che si intrecciano e si ricorrono. L'apparente sottomissione della donna orientale, la necessità per la donna occidentale di nascondere intelligenza e cultura, quella di esser magra a tutti i costi. E ancora l'ambientazione, il sogni, il ricorrere costante dei profumi dell' Arabia, del grande salone di marmo, della vasca, dei gelsomini.

Va precisato che per questi suoi testi la Monaco aveva pensato ad un'articolazione diversa. In effetti il copione in mio possesso vede al primo posto Interior design, al secondo Dormire, sognare, seguiti da Aragosta a colazione,Tutto in una notte e L'incubo della taglia 42. Ciò per dare maggiore ritmo e spezzare la monotonia monotematica dei copioni. In ogni caso sono costruiti con un equilibrato mix di rammarico, ironia e supremo menefreghismo.

Anche l'analisi di questi testi conferma quanto la Monaco sia stata influenzata da un aforisma di Nietzsche: “Un irresistibile impulso alla metamorfosi di se stesso e ad agire con altri corpi e altre anime... è questa la prima condizione di ogni arte drammatica; solo che prova questo impulso e questo incantesimo è drammaturgo". Per concludere l'intervento ribadisco che i suoi testi comici hanno avuto maggior successo per l'esigenza, molto forte nello spettatore, di svagarsi dimenticando per un attimo ciò che non va nella vita di tutti i giorni. In effetti, come sosteneva nel 1534 Rabelais nella 
lettera ai lettori del Gargantua," È meglio scrivere di riso che di lacrime perché il riso è proprio dell'uomo".

\section{RIFERIMENTI BIBLIOGRAFICI}

Battiflora, P., Maghreb, il fascino del mondo dai mille segreti, su "Il secolo XIX", 7 marzo 2002.

Piastra, R., Il teatro di Patrizia Monaco, inedita tesi di laurea ,a.a. 2006-2007.

Trovato, R., La scrittura come vita e come gioco. Il teatro di Patrizia Monaco in Las voces de las Diosas, Sevilla, Arcibel, 2012, pp. 1321-1345. 